\title{
A Vibration Energy Approach Used to Identify Temperature Trending in Railroad Tapered-Roller Bearings
}

\author{
Constantine M. Tarawneh \\ Department of Mechanical Engineering, College of Engineering and Computer Science, The University of Texas- \\ Pan American, Edinburg, TX 78539-2999, USA
}

\author{
Rafael K. Maldonado, Arturo A. Fuentes and Javier A. Kypuros \\ The University of Texas-Pan American, Edinburg, TX, USA
}

(Received 16 July 2014; accepted 5 March 2014)

Bearing temperature trending is a phenomenon that has plagued the railroad industry for decades and has resulted in costly train stoppages and non-verified bearing removals. Initial experimental studies conducted at The University of Texas-Pan American to explore this troubling phenomenon identified potential sources for the abrupt changes in temperature exhibited by some railroad bearings. The authors hypothesize that vibration-induced rollermisalignment is the root cause for bearing temperature trending. Hence, subsequent research focused on providing validation for the proposed hypothesis through vibration monitoring techniques. To that end, dynamic testers were used to run railroad bearings at the various speeds and loads that they experience in the field. A "trigger" bearing with a known cup raceway defect was used as a vibration source to induce roller misalignment on neighbouring defect-free bearings. Results show that the vibration energy of a bearing would decrease prior to an increase in temperature. In theory, misaligned rollers would vibrate less, leading to a decrease in the overall vibration energy, while also generating sufficient friction to account for the observed temperature increase. Typically, rollers realign themselves through geometrical thermal expansions or changes in the operating conditions, thus, returning to normal temperature and vibration levels. This paper outlines the research findings.

\section{NOMENCLATURE}

$B_{\mathrm{d}} \quad$ mean diameter of a roller

$D_{\text {cone }} \quad$ cone tapered large end diameter

$D_{1} \quad$ roller large end diameter

$D_{\text {roller }}$ roller mean diameter

$D_{\text {s }} \quad$ roller small end diameter

$L \quad$ cone raceway tapered length

$N_{\mathrm{b}} \quad$ number of rollers

$P_{\mathrm{d}} \quad$ pitch diameter (span between the centres of two opposite rolling elements)

$R_{\text {cone }} \quad$ radius of the cone

$R_{\text {cup }} \quad$ radius of the cup

$\alpha_{\text {roller }}$ roller apex angle

$\theta \quad$ contact angle between the cone raceway and tapered roller

$\phi_{\text {cone }} \quad$ cone raceway tapered angle

$\omega_{\text {cage }}$ fundamental frequency of the cage

$\omega_{\text {cone }}$ fundamental frequency of the cone

$\omega_{\text {in }} \quad$ fundamental frequency of a roller passing over a defect on the cone raceway

$\omega_{\mathrm{o}} \quad$ rotational frequency of the axle

$\omega_{\text {out }}$ fundamental frequency of a roller passing over a defect on the cup raceway

$\omega_{\text {roller }}$ fundamental frequency of the roller

$\omega_{\text {rolldef }}$ fundamental frequency of a roller defect as it contacts the cup and cone raceway

BPFI ball pass frequency inner race or cone

BPFO ball pass frequency outer race or cup

$\begin{array}{ll}\text { BSF } & \text { ball spin or roller/cage frequency } \\ \text { FTF } & \text { fundamental train/cage frequency } \\ \text { HBD } & \text { hot-box detector } \\ \text { PS } & \text { power spectrum } \\ \text { PSD } & \text { power spectral density }\end{array}$

\section{INTRODUCTION}

One of the main concerns troubling railroad bearing manufacturers is the unexplained abrupt increase in the operating temperature of a healthy (defect-free) tapered-roller bearinga phenomenon referred to in field service as bearing temperature trending. Currently, trended bearings are removed from service since they exhibit similar behaviour to a burn-off bearing at the end of its life. This troubling phenomenon has resulted in many costly delays associated with train stoppages, as well as false bearing removals by wayside temperature monitoring devices such as the Hot Box Detector (HBD). According to data collected by Amsted Rail from 2001 to 2007, an average of nearly $40 \%$ of bearing removals are non-verified. A non-verified bearing is one that, upon disassembly and inspection, is found not to exhibit any of the commonly documented causes of bearing failure such as: spalling, water contamination, loose bearings, broken components, lubrication, damaged seals, etc.

HBDs measure the infrared radiation emissions present in a railroad bearing and have been in place since the 1950's. The $\mathrm{HBD}$ is set to trigger an alert when a bearing is running $94.4^{\circ} \mathrm{C}$ $\left(170^{\circ} \mathrm{F}\right)$ above ambient conditions. However, a more recent approach adopted by some railroads utilizes the HBD data to compare each bearing's temperature to the average tempera- 